International Mathematical Forum, 1, 2006, no. 2, 67-76

\title{
ON STRUCTURE OF KS-SEMIGROUPS
}

\author{
Kyung Ho Kim \\ Department of Mathematics \\ Chungju National University \\ Chungju 380-702, Korea \\ ghkim@chungju.ac.kr
}

\begin{abstract}
In this paper, we introduce a new class algebras related to BCK-algebras and semigroups, called a KS-semigroup and define an ideal of a KS-semigroups and a strong KS-semigroup, and characterizations of ideals is given. Also we define a congruence relation on a KS-semigroups and a quotient KS-semigroups and prove the isomorphism.
\end{abstract}

Mathematics Subject Classification: 06F35, 03G25

Keywords: KS-semigroup, strong KS-semigroup, P-ideal, quotient KSsemigroup, homomorphism

\section{INTRODUCTION}

The notion of BCK-algebras was proposed by Y. Imai and K. Iséki in 1966. In the same year, K. Iséki introduced the notion of BCI-algebra which is a generalization of a BCK-algebra. For the general development of BCK/BCIalgebras, the ideal theory plays an important role. We introduce a new class algebras related to BCK-algebras and semigroups, called a KS-semigroup. In this paper, we define an ideal of a KS-semigroups and a strong KS-semigroup, and characterizations of ideals is given. Also we define a congruence relation on a KS-semigroups and a quotient KS-semigroups and prove the isomorphism.

\section{Preliminaries}

We review some definitions and properties that will be useful in our results.

A $B C I$-algebra is a triple $(X, *, 0)$, where $X$ is a nonempty set, "* " is a binary operation on $X, 0 \in H$ is an element such that the following four axioms are satisfied for every $x, y, z \in X$ : 
(I) $((x * y) *(x * z)) *(z * y)=0$,

(II) $(x *(x * y))) * y=0$,

(III) $x * x=0$,

(IV) $x * y=0, y * x=0$ implies $x=y$.

A BCI-algebra $X$ satisfying $0 * x=0$ for all $x \in X$ is called a $B C K$-algebra. If $X$ is a BCK-algebra, then the relation $x \leq y$ if and only if $x * y=0$ is a partial order on $X$, which will be called the natural ordering on $X$.

A BCK-algebra $X$ has the following properties for any $x, y, z \in X$ :

(1) $x * 0=x$,

(2) $(x * y) * z=(x * z) * y$,

(3) $x \leq y$ implies that $x * z \leq y * z$ and $z * y \leq z * x$,

(4) $(x * z) *(y * z) \leq x * y$,

A nonempty subset $I$ of a BCK/BCI-algebra is called an ideal if it satisfies

(i) $0 \in X$,

(ii) $x * y \in X$ and $y \in X$ imply $x \in X$ for all $x, y \in X$.

Any ideal $I$ has the property: $y \in I$ and $x \leq y$ imply $x \in I$.

For a BCK-algebra $X$, the set $X_{+}:=\{x \in X \mid 0 \leq x\}$ is called the BCK-part of $X$. If $X_{+}=\{0\}$, then we say that $X$ is a $p$-semisimple BCI-algebra. Note that a BCI-algebra $X$ is p-semisimple if and only if $0 *(0 * x)=x$ for all $x \in X$.

An KS-semigroup is a non-empty set $X$ with two binary operation " $*$ " and "." and constant 0 satisfying the axioms:

$(\mathrm{V})(X, *, 0)$ is a BCK-algebra,

(VI) $(X, \cdot)$ is a semigroup,

(VII) The operation "." is distributive (on both sides) over the operation "*", that is, $x \cdot(y * z)=(x \cdot y) *(x \cdot z)$ and $(x * y) \cdot z=(x \cdot z) *(y \cdot z)$ for all $x, y, z \in X$.

We shall write the multiplication $x \cdot y$ by $x y$ for convenience.

Example 2.1. Let $X=\{0, a, b, c, d\}$. Define $*$-operation and multiplication "." by the following tables

\begin{tabular}{c|ccccc}
$*$ & 0 & $a$ & $b$ & $c$ & $d$ \\
\hline 0 & 0 & 0 & 0 & 0 & 0 \\
$a$ & $a$ & 0 & $a$ & $a$ & 0 \\
$b$ & $b$ & $b$ & 0 & 0 & 0 \\
$c$ & $c$ & $c$ & $c$ & 0 & 0 \\
$d$ & $d$ & $d$ & $d$ & $d$ & 0
\end{tabular}

\begin{tabular}{c|ccccc}
$\cdot$ & 0 & $a$ & $b$ & $c$ & $d$ \\
\hline 0 & 0 & 0 & 0 & 0 & 0 \\
$a$ & 0 & 0 & 0 & 0 & 0 \\
$b$ & 0 & 0 & 0 & 0 & $b$ \\
$c$ & 0 & 0 & 0 & $b$ & $c$ \\
$d$ & 0 & $a$ & $b$ & $c$ & $d$
\end{tabular}

Then, by routine calculations, we can see that $X$ is an $K S$-semigroup.

Lemma 2.2. Let $X$ be an KS-semigroup. Then we have

(i) $0 x=x 0=0$,

(2) $x \leq y$ implies that $x z \leq y z$ and $z x \leq z y$, for all $x, y, z \in X$. 
A non-empty subset $A$ of a semigroup $(X, \cdot)$ is said to be left (resp. right) stable if $x a \in A$ (resp. $a x \in A$ ) whenever $x \in X$ and $a \in A$. Both left and right stable is two-sided stable or simply stable.

Definition 2.3. A non-empty subset $A$ of a KS-semigroup $X$ is called a left(resp. right) ideal of $X$ if

(i) $A$ is a left(resp. right) stable subset of $(X, \cdot)$,

(ii) for any $x, y \in X, x * y \in A$ and $y \in A$ imply that $x \in A$.

A both of left and right ideal is called a two-sided ideal or simply an ideal. Note that $\{0\}$ and $X$ are ideals. If $A$ is a left(resp. right) ideal of an KSsemigroup of $X$, then $0 \in A$. Thus $A$ is an ideal of $X$.

Example 2.4. Let $X=\{0, a, b, c\}$. Define *-operation and multiplication "." by the following tables

\begin{tabular}{c|ccccc|cccc}
$*$ & 0 & $a$ & $b$ & $c$ & $\cdot$ & 0 & $a$ & $b$ & $c$ \\
\hline 0 & 0 & 0 & 0 & 0 & 0 & 0 & 0 & 0 & 0 \\
$a$ & $a$ & 0 & $a$ & 0 & $a$ & 0 & $a$ & 0 & $a$ \\
$b$ & $b$ & $b$ & 0 & 0 & $b$ & 0 & 0 & $b$ & $b$ \\
$c$ & $c$ & $b$ & $a$ & 0 & $c$ & 0 & $a$ & $b$ & $c$
\end{tabular}

Then, by routine calculations, we can see that $X$ is an KS-semigroup. If $A=\{0, a\}$, then $A$ is an ideal of a KS-semigroup $X$.

Definition 2.5. An ideal $A$ of an KS-semigroup $X$ is said to be closed if $x \in X$ implies $0 * x \in A$.

\section{MAIN RESUlts}

In what follows, let $X$ denote a KS-semigroup unless otherwise specified.

Definition 3.1. A non-empty subset $A$ of a KS-semigroup $X$ is called a left(resp. right) $\mathrm{P}$-ideal of $X$ if

(i) $A$ is a left(resp. right) stable subset of $(X, \cdot)$,

(ii) for any $x, y, z \in X,(x * y) * z \in A$ and $y * z \in A$ imply that $x * z \in A$.

Example 3.2. Let $X=\{0, a, b, c\}$. Define *-operation and multiplication "." by the following tables

\begin{tabular}{l|lllll|llll}
$*$ & 0 & $a$ & $b$ & $c$ & $\cdot$ & 0 & $a$ & $b$ & $c$ \\
\hline 0 & 0 & 0 & 0 & 0 & 0 & 0 & 0 & 0 & 0 \\
$a$ & $a$ & 0 & $a$ & $a$ & $a$ & 0 & $a$ & 0 & 0 \\
$b$ & $b$ & $b$ & 0 & $b$ & $b$ & 0 & 0 & $b$ & 0 \\
$c$ & $c$ & $c$ & $c$ & 0 & $c$ & 0 & $c$ & 0 & 0
\end{tabular}

Then, by routine calculations, we can see that $X$ is an KS-semigroup. Let $A=\{0, a\}$. Then $A$ is a $P$-ideal of a KS-semigroup $X$.

Example 3.3. Let $X=\{0, a, b, c\}$. Define *-operation and multiplication "." by the following tables 


\begin{tabular}{c|cccc}
$*$ & 0 & $a$ & $b$ & $c$ \\
\hline 0 & 0 & 0 & 0 & 0 \\
$a$ & $a$ & 0 & 0 & $a$ \\
$b$ & $b$ & $a$ & 0 & $b$ \\
$c$ & $c$ & $c$ & $c$ & 0
\end{tabular}

\begin{tabular}{c|cccc}
$\cdot$ & 0 & $a$ & $b$ & $c$ \\
\hline 0 & 0 & 0 & 0 & 0 \\
$a$ & 0 & 0 & 0 & $a$ \\
$b$ & 0 & 0 & 0 & $b$ \\
$c$ & 0 & 0 & 0 & $c$
\end{tabular}

Then, by routine calculations, we can see that $X$ is an KS-semigroup. Let $A=\{0, a\}$. Then $A$ is a $\mathrm{P}$-ideal of a KS-semigroup $X$.

Theorem 3.4. Every P-ideal of a KS-semigroup $X$ is an ideal but the converse is not true.

Proof. Suppose that $A$ is a $\mathrm{P}$-ideal of a

KS-semigroup. Let $x, y \in X$ be such that $x * y \in A$ and $y \in A$. It follows from (1) that

$$
(x * y) * 0=x * y \in A \text { and } y * 0=y \in A .
$$

Therefore, by Definition 3.1(ii), we obtain $x=x * 0 \in A$. So, $A$ is an ideal of $X$. In Example 3.2, $\{0, b\}$ is an ideal but not a P-ideal of $X$ because $(a * c) * b=$ $a * b=0 \in\{0, b\}$ but $c * b=c \notin\{0, b\}$.

Definition 3.5. A strong KS-semigroup is a KS-semigroup $X$ satisfying $x *$ $y=x * x y$ for each $x, y \in X$.

Example 3.6. In Example 2.4, we are easy to prove that $(X, *, \cdot, 0)$ is a strong KS-semigroup.

Lemma 3.7. Let $X$ be a strong KS-semigroup. Then

(i) $x y * y=0$ for all $x, y \in X$,

(ii) $x * y=0$ if and only if $x * x y=0$ for any $x, y \in X$.

Proof. (1) For any $x, y \in X, x y * y=x y *(x y) y=x y * x(y y)=x(y * y y)=$ $x(y * y)=0$.

(2) It is easy to show from the definition of strong KS-semigroup and the above (1).

The element 1 is called a unity in a KS-semigroup $X$ if $1 x=x 1=x$ for all $x \in X$. If $X$ is a strong KS-semigroup with a unity 1 , then 1 is the greatest element in $X$ since $x * 1=x * x 1=x * x=0$ for all $x \in X$.

Theorem 3.8. Let $X$ be a strong KS-semigroup with a unity 1 and $A$ any non-empty subset of $X$. If $y \in A$ and $x \leq y$ imply $x \in A$, then $A$ is an ideal of $X$.

Proof. Suppose that $y \in A$ and $x \leq y$ imply $x \in A$. If $x, y \in A$, then $x * y \in A$, since $x * y=x * x y=x(1 * y) \leq x 1=x \in A$. Next, let $s \in X$ and $a \in A$. Then

$$
a s * a=a(s * 1)=a 0=0
$$


and

$$
s a * a=(s * 1) a=0 a=0,
$$

hence $a s \leq a$ and $s a \leq a$, that is, $A X \subseteq A$ and $X A \subseteq A$. It follows that $A$ is an ideal of $X$.

For any $x, y$ in $X$, denote $x \wedge y=y *(y * x)$. Obviously, $x \wedge y$ is a lower bound of $x$ and $y$, and $x \wedge x=x, x \wedge 0=0 \wedge x=0$.

Theorem 3.9. Let $X$ be a strong KS-semigroup satisfying $x *(x * y)=y *(y * x)$. Then we have

$$
x \wedge y=x y
$$

Proof. For any $x, y \in X$, we have

$$
\begin{aligned}
x \wedge y=x *(x * y) & =x *(x * x y)=x y *(x y * x) \\
& =x y * x(y * 1)=x y * x 0 \\
& =x y * 0=x y
\end{aligned}
$$

Proposition 3.10. Let $X$ be a strong KS-semigroup with 1 and satisfying $x *(x * y)=y *(y * x)$. Then the set

$$
\operatorname{ann}(a)=\{x \in X \mid x \wedge a=0, a \in X\}
$$

is an ideal of $X$.

Proof. Let $y \in \operatorname{ann}(a)$ and $x * y \in \operatorname{ann}(a)$. Then we have $y \wedge a=y a=0$, and $x a=x a * 0=x a * y a \in \operatorname{ann}(a)$. Hence we get $x \in \operatorname{ann}(a)$. Also, let $x \in \operatorname{ann}(a)$ and $s \in X$. Then we obtain $x \wedge a=x a=0$, and so, $s x \wedge a=$ $(s x) a=s(x a)=s 0=0$. Thus $s x \in \operatorname{ann}(a)$. Similarly, we have $x s \in \operatorname{ann}(a)$. This completes the proof.

Let $X$ be a strong KS-semigroup satisfying $x *(x * y)=y *(y * x)$. If $s \leq t$ for all $s, t \in X$, then we have $\operatorname{ann}(s) \subseteq$ ann $(t)$.

Definition 3.11. Let $X$ be a KS-semigroup and let $\rho$ be a binary relation on $X$. Then

(1) $\rho$ is said to be right (resp. left) compatible if whenever $(x, y) \in \rho$ then $(x * z, y * z) \in \rho(\operatorname{resp} .(z * x, z * y) \in \rho)$ and $(x z, y z) \in \rho(\operatorname{resp} .(z x, z y) \in \rho)$ for all $x, y, z \in X$;

(2) $\rho$ is said to be compatible if $(x, y) \in \rho$ and $(u, v) \in \rho$ imply $(x * u, y * v) \in \rho$ and $(x u, y v) \in \rho$ for all $x, y, u, v \in X$,

(3) A compatible equivalence relation is called a congruence relation.

Using the notion of left (resp. right) compatible relation, we give a characterization of a congruence relation.

Theorem 3.12. Let $X$ be a KS-semigroup. Then an equivalence relation $\rho$ on $X$ is congruence if and only if it is both left and right compatible. 
Proof. Assume that $\rho$ is a congruence relation on $X$. Let $x, y \in X$ be such that $(x, y) \in \rho$. Note that $(z, z) \in \rho$ for all $z \in X$ because $\rho$ is reflexive. It follows from the compatibility of $\rho$ that $(x * z, y * z) \in \rho$ and $(x z, y z) \in \rho$. Hence $\rho$ is right compatible. Similarly, $\rho$ is left compatible.

Conversely, suppose that $\rho$ is both left and right compatible. Let $x, y, u, v \in$ $X$ be such that $(x, y) \in \rho$ and $(u, v) \in \rho$. Then $(x * u, y * u) \in \rho$ and $(x u, y u) \in \rho$. by the right compatibility. Using the left compatibility of $\rho$, we have $(y * u, y *$ $v) \in \rho$ and $(y u, y v) \in \rho$. It follows from the transitivity of $\rho$ that $(x * u, y * v) \in \rho$ and $(x u, y v) \in \rho$. Hence $\rho$ is congruence.

For a binary relation $\rho$ on a KS-semigroup $X$, we denote

$$
x \rho:=\{y \in X \mid(x, y) \in \rho\} \text { and } X / \rho:=\{x \rho \mid x \in X\} .
$$

Theorem 3.13. Let $\rho$ be a congruence relation on a KS-semigroup $X$. Then $X / \rho$ is a KS-semigroup under the operations

$$
x \rho * y \rho=(x * y) \rho \text { and }(x \rho)(y \rho)=(x y) \rho
$$

for all $x \rho, y \rho \in X / \rho$.

Proof. Since $\rho$ is a congruence relation, the operations are well-defined. Clearly, $(X / \rho, *)$ is a BCK- algebra and $(X / \rho, \cdot)$ is a semigroup. For every $x \rho, y \rho, z \rho \in X / \rho$, we have

$$
\begin{aligned}
x \rho(y \rho * z \rho) & =x \rho(y * z) \rho=x(y * z) \rho \\
& =(x y * x z) \rho=(x y) \rho *(x z) \rho \\
& =x \rho y \rho * x \rho z \rho,
\end{aligned}
$$

and

$$
\begin{aligned}
(x \rho * y \rho) z \rho & =(x * y) \rho z \rho=((x * y) z) \rho \\
& =(x z * y z) \rho=(x z) \rho *(y z) \rho \\
& =x \rho z \rho * y \rho z \rho .
\end{aligned}
$$

Thus $X / \rho$ is a KS-semigroup.

Definition 3.14. Let $X$ and $X^{\prime}$ be KS-semigroups. A mapping $f: X \rightarrow X^{\prime}$ is called a KS-semigroup homomorphism (briefly, homomorphism) if $f(x * y)=$ $f(x) * f(y)$ and $f(x y)=f(x) f(y)$ for all $x, y \in X$.

Let $f: X \rightarrow Y$ be a homomorphism of KS-semigroup. Then the set $\{x \in$ $X \mid f(x)=0\}$ is called the kernel of $f$, and denote by ker $f$. Moreover, the set $\{f(x) \in Y \mid x \in X\}$ is called the image of $f$, and denote by $i m f$.

Lemma 3.15. Let $f: X \rightarrow X^{\prime}$ be a KS-semigroup homomorphism. Then

(1) $f(0)=0$,

(2) $x \leq y$ imply $f(x) \leq f(y)$.

(3) $f(x \wedge y)=f(x) \wedge f(y)$.

Proof. (1). Suppose that $x$ is an element of $X$. Then

$$
f(0)=f(x * x)=f(x) * f(x)=0
$$


(2) Let $x \leq y$. Then we have $x * y=0$. Thus we have

$$
0=f(x * y)=f(x) * f(y)
$$

and so $f(x) \leq f(y)$.

(3) $f(x \wedge y)=f(y *(y * x))=f(y) *(f(y) * f(x))=f(x) \wedge f(y)$.

Proposition 3.16. Let $f: X \rightarrow X^{\prime}$ be a KS-semigroup homomorphism and $J=f^{-1}(0)=\{0\}$. Then $f(x) \leq f(y)$ imply $x \leq y$.

Proof. If $f(x) \leq f(y)$, then we have $f(x) * f(y)=f(x * y)=0$, and so $x * y$ is an element of $J$. Hence $x * y=0$, and so we obtain $x \leq y$.

Theorem 3.17. Let $\rho$ be a congruence relation on a KS-semigroup $X$. Then the mapping $\rho^{*}: X \rightarrow X / \rho$ defined by $\rho^{*}(x)=x \rho$ for all $x \in X$ is a KSsemigroup homomorphism.

Proof. Let $x, y \in X$. Then $\rho^{*}(x * y)=(x * y) \rho=x \rho * y \rho=\rho^{*}(x) * \rho^{*}(y)$, and $\rho^{*}(x y)=(x y) \rho=(x \rho)(y \rho)=\rho^{*}(x) \rho^{*}(y)$. Hence $\rho^{*}$ is a KS-semigroup homomorphism.

Theorem 3.18. Let $X$ and $X^{\prime}$ be KS-semigroups and let $f: X \rightarrow X^{\prime}$ be a KS-semigroup homomorphism. Then the set

$$
K_{f}:=\{(x, y) \in X \times X \mid f(x)=f(y)\}
$$

is a congruence relation on $X$ and there exists a unique 1-1 KS-semigroup homomorphism $\bar{f}: X / K_{f} \rightarrow X^{\prime}$ such that $\bar{f} \circ K_{f}^{*}=f$, where $K_{f}^{*}: X \rightarrow X / K_{f}$. That is, the following diagram commute:

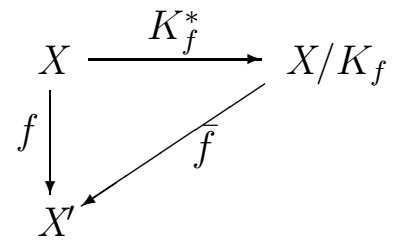

Proof. It is clear that $K_{f}$ is an equivalence relation on $X$. Let $x, y, u, v \in X$ be such that $(x, y),(u, v) \in K_{f}$. Then $f(x)=f(y)$ and $f(u)=f(v)$, which imply that

$$
f(x * u)=f(x) * f(u)=f(y) * f(v)=f(y * v)
$$

and

$$
f(x u)=f(x) f(u)=f(y) f(v)=f(y v) .
$$

It follows that $(x * u, y * v) \in K_{f}$ and $(x u, y v) \in K_{f}$. Hence $K_{f}$ is a congruence relation on $X$. Let $\bar{f}: X / K_{f} \rightarrow X^{\prime}$ be a map defined by $\bar{f}\left(x K_{f}\right)=f(x)$ for all $x \in X$. It is clear that $\bar{f}$ is well-defined. For any $x K_{f}, y K_{f} \in X / K_{f}$, we have

$$
\begin{aligned}
\bar{f}\left(x K_{f} * y K_{f}\right) & =\bar{f}\left((x * y) K_{f}\right)=f(x * y) \\
& =f(x) * f(y)=\bar{f}\left(x K_{f}\right) * \bar{f}\left(y K_{f}\right)
\end{aligned}
$$


and

$$
\begin{aligned}
\bar{f}\left(\left(x K_{f}\right)\left(y K_{f}\right)\right) & =\bar{f}\left((x y) K_{f}\right)=f(x y) \\
& =f(x) f(y)=\bar{f}\left(x K_{f}\right) \bar{f}\left(y K_{f}\right) .
\end{aligned}
$$

If $\bar{f}\left(x K_{f}\right)=\bar{f}\left(y K_{f}\right)$, then $f(x)=f(y)$ and so $(x, y) \in K_{f}$, that is, $x K_{f}=y K_{f}$. Thus $\bar{f}$ is a 1-1 KS-semigroup homomorphism. Now let $g$ be a KS-semigroup homomorphism from $X / K_{f}$ to $X^{\prime}$ such that $g \circ K_{f}^{*}=f$. Then

$$
g\left(x K_{f}\right)=g\left(K_{f}^{*}(x)\right)=f(x)=\bar{f}\left(x K_{f}\right)
$$

for all $x K_{f} \in X / K_{f}$. It follows that $g=\bar{f}$ so that $\bar{f}$ is unique. This completes the proof.

Corollary 3.19. Let $\rho$ and $\sigma$ be congruence relations on a KS-semigroup $X$ such that $\rho \subseteq \sigma$. Then the set

$$
\sigma / \rho:=\{(x \rho, y \rho) \in X / \rho \times X / \rho \mid(x, y) \in \sigma\}
$$

is a congruence relation on $X / \rho$ and there exists a 1-1 and onto KS-semigroup homomorphism from $\frac{X / \rho}{\sigma / \rho}$ to $X / \sigma$.

Proof. Let $g: X / \rho \rightarrow X / \sigma$ be a function defined by $g(x \rho)=x \sigma$ for all $x \rho \in X / \rho$. Since $\rho \subseteq \sigma$, it follows that $g$ is a well-defined onto KSsemigroup homomorphism. According to Theorem 3.18, it is sufficient to show that $K_{g}=\sigma / \rho$. Let $(x \rho, y \rho) \in K_{g}$. Then $x \sigma=g(x \rho)=g(y \rho)=y \sigma$ and so $(x, y) \in \sigma$. Hence $(x \rho, y \rho) \in \sigma / \rho$, and thus $K_{g} \subseteq \sigma / \rho$.

Conversely, if $(x \rho, y \rho) \in \sigma / \rho$, then $(x, y) \in \sigma$ and so $x \sigma=y \sigma$. It follows that

$$
g(x \rho)=x \sigma=y \sigma=g(y \rho)
$$

so that $(x \rho, y \rho) \in K_{g}$. Hence $K_{g}=\sigma / \rho$, and the proof is complete.

Theorem 3.20. Let $I$ be an ideal of a KS-semigroup $X$. Then $\rho_{I}:=(I \times I) \cup$ $\Delta_{X}$ is a congruence relation on $X$, where $\Delta_{X}:=\{(x, x) \mid x \in X\}$.

Proof. Clearly, $\rho_{I}$ is reflexive and symmetric. Noticing that $(x, y) \in \rho_{I}$ if and only if $x, y \in I$ or $x=y$, we know that if $(x, y) \in \rho_{I}$ and $(y, z) \in \rho_{I}$ then $(x, z) \in \rho_{I}$. Hence $\rho_{I}$ is an equivalence relation on $X$. Assume that $(x, y) \in \rho_{I}$ and $(u, v) \in \rho_{I}$. Then we have the following four cases: (i) $x, y \in I$ and $u, v \in I$; (ii) $x, y \in I$ and $u=v$; (iii) $x=y$ and $u, v \in I$; and (iv) $x=y$ and $u=v$. In either case, we get $x * u=y * v$ or $(x * u, y * v) \in I \times I$, and $x u=y v$ or $(x u, y v) \in I \times I$. Therefore $\rho_{I}$ is a congruence relation on $X$.

Theorem 3.21. Let $f: X \rightarrow Y$ be a homomorphism of a KS-semigroup. Then $\operatorname{ker} f$ is an ideal of $X$.

Proof. Let $x * y \in \operatorname{ker} f$ and $y \in \operatorname{ker} f$. Then $0=f(x * y)=f(x) * f(y)=$ $f(x) * 0=f(x)$. Hence we have $x \in \operatorname{ker} f$. Let $x \in X$ and $a \in \operatorname{ker} f$. Then we 
obtain $f(a x)=f(a) f(x)=0 f(a)=0$ and $f(x a)=f(x) f(a)=f(x) 0=0$. Hence $a x, x a \in \operatorname{ker} f$. This completes the proof.

Let $A$ be an ideal of a KS-semigroup $X$. We define a relation " " on $X$ as follows

$$
x \underset{A}{\sim} y \text { if and only if } x * y \in A \text { and } y * x \in A .
$$

Then " $\sim$ " is a congruence relation on $X$.

Let $X$ be a KS-semigroup and denote by $A_{x}$ the equivalence class containing $x \in X$, and by $X / A$ the set of all equivalence classes of $X$ with respect to " $\sim$ ", that is,

$$
A_{x}:=\{y \in X \mid x \underset{A}{\sim} y\} \text { and } X / A:=\left\{A_{x} \mid x \in X\right\} .
$$

Note that $A_{x}=A_{y}$ if and only if $x \underset{A}{\sim} y$, and $A=A_{0}$.

Theorem 3.22. If $A$ is an ideal of a KS-semigroup $X$, then mapping $\psi: X \rightarrow$ $X / A$ given by $\psi(x)=A_{x}$ is an epimorphism with kernel $A$.

Proof. The map $\psi: X \rightarrow X / A$ is clearly surjective and since $\psi(x * y)=$ $A_{x * y}=A_{x} * A_{y}=\psi(x) \otimes \psi(y)$ and $\psi(x y)=A_{x y}=A_{x} \odot A_{y}=\psi(x) \odot \psi(y), \psi$ is an epimorphism. Now $\operatorname{ker} \psi=\left\{x \in X \mid \psi(x)=A_{x}=A_{0}\right\}=\{x \in X \mid x \in$ $A\}=A_{0}$.

Theorem 3.23. If $A$ is an ideal of a KS-semigroup $X$, then $\left(X / A, \otimes, \odot, A_{0}\right)$ is an KS-semigroup under the binary operations

$$
A_{x} \otimes A_{y}=A_{x * y} \text { and } A_{x} \odot A_{y}=A_{x y}
$$

for all $A_{x}, A_{y} \in X / A$.

Proof. We are easy to prove that $\left(X / A, \otimes, \odot, A_{0}\right)$ is a BCK-algebra. First we show that $\odot$ is well-defined. Let $A_{x}=A_{u}$ and $A_{y}=A_{v}$. Then we have $x y * x v=x(y * v) \in A$ and $x v * x y=x(v * y) \in A$. Thus $x y \sim x v$. On the other hand, $x v * u v=(x * u) v \in A$ and $u v * x v=(u * x) v \in A$. Hence, $x v \underset{A}{\sim} u v$, and so $A_{x y}=A_{u v}$. Therefore, $(X / A, \odot)$ is a semigroup. Moreover, for any $A_{x}, A_{y}, A_{z} \in X / A$, we obtain $A_{x} \odot\left(A_{y} \otimes A_{z}\right)=A_{x} \odot A_{y * z}=A_{x(y * z)}=$ $A_{x y * x z}=A_{x y} \otimes A_{x z}=\left(A_{x} \odot A_{y}\right) \otimes\left(A_{x} \odot A_{z}\right)$. Similarly, we get $\left(A_{x} \otimes A_{y}\right) \odot A_{z}=$ $\left(A_{x} \odot A_{z}\right) \otimes\left(A_{y} \odot A_{z}\right)$. Therefore, $X / A$ is a KS-semigroup.

Theorem 3.24. Let $f: X \rightarrow Y$ be a homomorphism of KS-semigroups. Then ker $f$ is an ideal of $X$.

Proof. Let $x * y \in \operatorname{ker} f$ and $y \in \operatorname{ker} f$. Then $0=f(x * y)=f(x) * f(y)=$ $f(x) * 0=f(x)$. Hence we have $x \in$ ker $f$. Let $x \in X$ and $a \in k e r f$. Then by Lemma 2.2, we obtain $f(a x)=f(a) f(x)=0 f(a)=0$ and $f(x a)=f(x) f(a)=$ $f(x) 0=0$. Hence $x a, a x \in \operatorname{ker} f$.

Theorem 3.25. Let $f: X \rightarrow Y$ be a homomorphism of a KS-semigroup. Then for any ideal of $X, A /(\operatorname{ker} f \cap A)=f(A)$. 
Proof. Clearly, ker $f \cap A$ is an ideal of $A$. Let $B=\operatorname{ker} f \cap A$. We define a mapping $\psi: A / B \rightarrow Y$ with $\psi\left(B_{x}\right)=f(x)$ where $x \in A$. Then for any $B_{x}, B_{y} \in A / B$, we have

$$
\begin{aligned}
B_{x}=B_{y} & \Leftrightarrow x * y \in B, y * x \in B, \\
& \Leftrightarrow f(x * y)=0, f(y * x)=0, \\
& \Leftrightarrow f(x) * f(y)=0, f(y) * f(x)=0, \\
& \Leftrightarrow f(x)=f(y), \\
& \Leftrightarrow \psi\left(B_{x}\right)=\psi\left(B_{y}\right) .
\end{aligned}
$$

Hence, $\psi$ is well-defined and one to one. Moreover, for all $B_{x}, B_{y} \in A / B$, we get

$$
\psi\left(B_{x} \otimes B_{y}\right)=\psi\left(B_{x * y}\right)=f(x * y)=f(x) * f(y)=\psi\left(B_{x}\right) * \psi\left(B_{y}\right),
$$

and

$$
\psi\left(B_{x} \odot B_{y}\right)=\psi\left(B_{x y}\right)=f(x y)=f(x) f(y)=\psi\left(B_{x}\right) \psi\left(B_{y}\right) .
$$

So $\psi$ is a homomorphism of a KS-semigroup. Thus we obtain $i m \psi=\left\{\psi\left(B_{x}\right) \mid\right.$ $x \in A\}=\{f(x) \mid x \in A\}=f(A)$. Therefore $A /(\operatorname{ker} f \cap A)=f(A)$.

Corollary 3.26. (First Isomorphism Theorem) If $f: X \rightarrow Y$ is a surjective homomorphism of a KS-semigroup, then $X / \operatorname{ker} f$ is isomorphic to $Y$.

\section{REFERENCES}

[1] M.Aslam and A. B. Thaheem, A note on p-semisimple BCI-algebras, Math. Japon 36 , (1991), 39-45.

[2] Z. Ghen and H. Wang, Closed ideals and congruences on BCI-algebras, Kobe J. Math 8, (1991), 1-9.

[3] K. Isėki, An algebra related with a propositional calculus, Proc. Japan. Acad 42 , (1966), 351-366.

[4] K. Isèki and S. Tanaka, An introduction to the theory of BCK-algebras, Math. Japon $23,(1969), 1-26$.

[5] T. D. Lei and C. C. Xi, p-radical in BCI-algebras, Math. Japon 30 , (1985), 511-517.

[6] J. K. Park, W. H. Shim and E. H. Roh, On isomorphim theorems in IS-algebras, Soochow Journal of Mathematics 27 (No. 2) , (2001), 153-160.

\section{Received: June 9, 2005}

